\title{
Dispenser Printed Electroluminescent Lamps on Textiles for Smart Fabric Applications
}

\author{
Marc de Vos, Russel Torah, John Tudor \\ University of Southampton \\ Building 59, University of Southampton, Southampton, \\ Hampshire, SO17 1BJ, United Kingdom
}

\begin{abstract}
Flexible electroluminescent (EL) lamps are fabricated onto woven textiles using a novel dispenser printing process. Dispenser printing utilises pressurised air to deposit ink onto a substrate through a syringe and nozzle. This work demonstrates the first use of this technology to fabricate EL lamps. The luminance of the dispenser printed EL lamps is compared to screen-printed EL lamps, both printed on textile, and also commercial EL lamps on polyurethane film. The dispenser printed lamps are shown to have a 1.5 times higher luminance than the best performing commercially available lamp, and have a comparable performance to the screen-printed lamps.
\end{abstract}

\section{1) Introduction}

Smart fabrics, also known as e-textiles, are "textiles that are able to sense stimuli from the environment, to react to them and adapt to them by integration of functionalities in the textile structure"'[1]. Electroluminescent lamps form part of the smart fabric described earlier, as they enable fabrics to react to stimuli by providing a controllable light source. Fabric is attractive as a substrate as it has formed a fundamental part of our lives for hundreds of years. If electronic functions can be successfully integrated in fabric, it offers the opportunity to add a second high value function to the fabrics primary function. Incorporating typical printed circuit boards (PCBs) onto fabrics using traditional processing techniques is impossible due to current harsh chemical PCB processes being incompatible with the majority of fabrics.

An important function in electronics is communicating information to the user; to fulfil this requirement light emitting displays are often used. A proposed solution to incorporate light emitting displays into smart fabrics utilises electroluminescent (EL) displays. EL lamps are visible in mobile phone keypads, watches, aeronautical and automotive instrumentation, and advertising panels [2]. Since the initial discovery of electroluminescence in 1936 [3] electroluminescent devices have been developed based on inorganic thin-film alternating-current EL (TFACEL) [4], inorganic semiconductor based EL (such as light emitting diodes) [5], and organic semiconductor EL (such as organic light emitting diodes) [6]. For the purposes of this research the term 'EL lamp' will be used to describe AC powder EL lamps such as those based on $\mathrm{ZnS}$ phosphors [3].

The attraction of EL displays for smart fabrics is primarily their simple device structure and their processing tolerances compared to OLEDs. During fabrication EL devices require no control of the surrounding atmosphere unlike OLEDs, and after fabrication they are very durable [7], as demonstrated by recent stretchable and deformable devices [8]. They also offer new possibilities for fabric decoration in wearable technology and architecture, creating interest from clothing designers and architects.

The ability to print electronics directly onto fabric is appealing as printing processes are often already used in fabric production. Printing technologies such as inkjet [9] and screen-printing [10] have been used in the past for smart fabric fabrication. This research focuses on a new fabrication technique known as dispenser printing [11]. Dispenser printing combines the digital printing of inkjet with the functional performance of screen-printed inks. The work demonstrates the first dispenser printed EL lamp on fabric, demonstrating the capability of the fabrication technology.

The paper will give a description of the dispenser printing process and compare it to screen-printing. The structure of a printed EL lamp is then shown, with a description of the layers and materials used for each layer. The results are presented showing the layer by layer print process, and the finished EL lamp. The layer thickness, luminance, and colour co-ordinates are measured and compared to that of a screen-printed EL lamp. Finally, the lamps are compared to a range of commercially available products and conclusions are drawn as to the capabilities of dispenser printing to fabricate EL lamps.

\section{2) Dispenser Printing Technique}

Dispenser printing is an additive digital manufacturing process offering the ability to print a wide range of inks and easily change the design. A dispenser printer operates by forcing ink stored in a syringe through a nozzle using a pulse of high pressure air. The dispenser printer is able to print a wide range of materials, due to the ability to select a suitable nozzle diameter to suit the maximum particle size in the ink and the ink viscosity (1-300 Pa.s). This capability provides an advantage over other direct write technologies such as inkjet which can operate with a limited range on ink particle sizes $(<200 \mathrm{~nm})$ and viscosities $(<0.02$ Pa.s $)$ [12]. The limited 


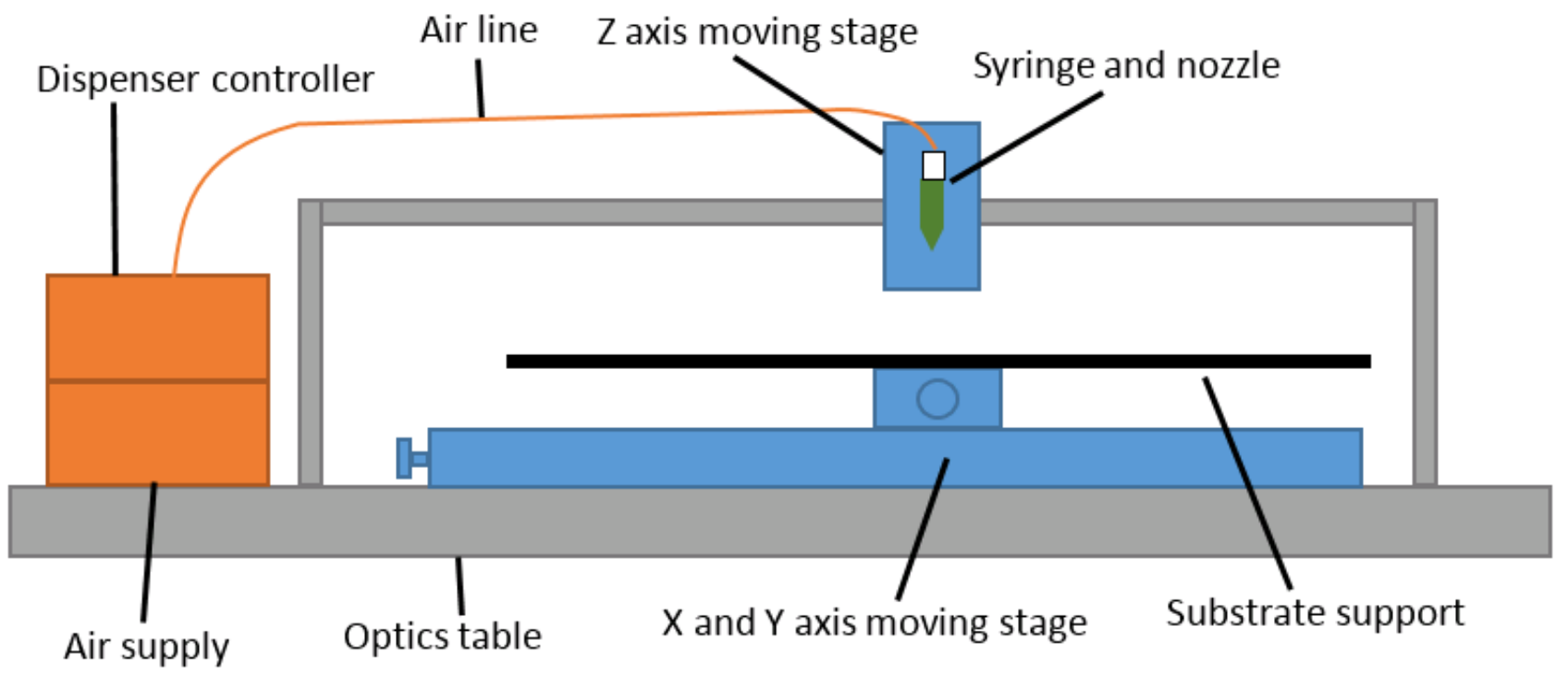

Figure 2. Diagram showing the components in a typical dispenser printer.

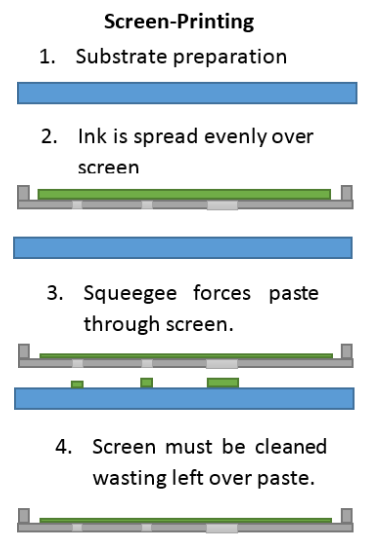

Figure 1. Diagram showing process differences between screen and dispenser printing.

particle size has been shown to make inkjet printing incompatible with most EL lamp phosphor particles as they are typically $20-40 \mu \mathrm{m}$, far in excess of the inkjet nozzle size[13].

The printed design is defined directly from a computer. This ability to change the design rapidly is also appealing in prototyping applications or one off designs allowing designs that were typically screen-printed to be quickly tested and altered. As a rapid prototyping process, dispenser printing reduces production time as it is a direct write technology; ink is only deposited in the areas required. Direct write technology also reduces environmental impact due to reduced waste and allows printing on complex geometries such as curved moulded plastic as it is non-contact. Other technologies such as screen-printing are better suited to mass-production as they are faster at producing multiple copies of the same design once the screens have been produced.

The inks used in dispenser printing are typically compatible with industrial mass production screen-printing processes. Therefore it is known that any prototype printed with the dispenser can be mass produced by screen-printing once the design is finalised, as the same inks are used for both

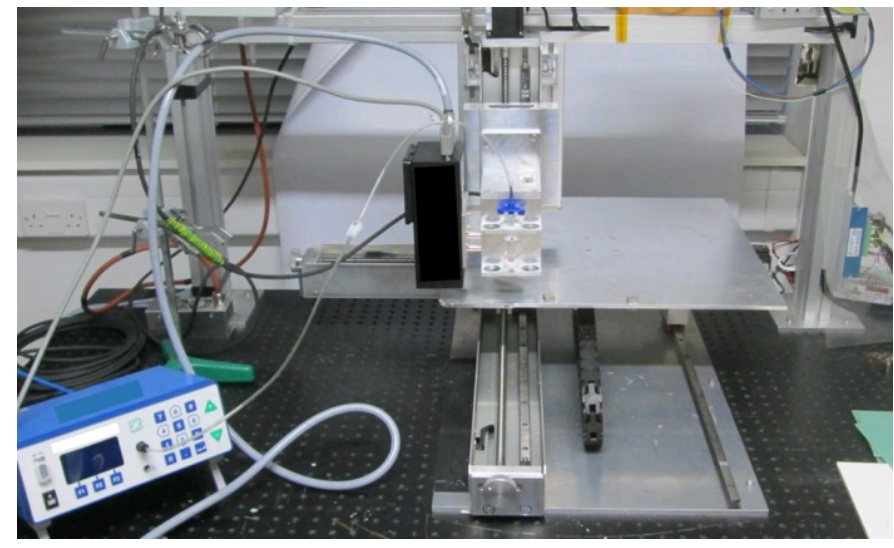

Figure 3. Image showing the University of Southampton dispenser printer.

processes. This is an advantage over inkjet printing where the inks require reformulating to be compatible with mass production screen-printing techniques.

Using dispenser printing to realise EL lamps offers the ability to print all the required layers directly onto fabric using a single printing technology. The dispensed materials, known as 'inks' to differentiate them from screen-printing pastes, are mostly functional particles dispersed in a binder to make them suitable for printing. However, screen-printable pastes can also be dispenser printed. Figure 1 highlights the process differences between dispenser and screen-printing for the example of a conductive track. The advantages make dispenser printing attractive to industries where ink costs are high or where the print design often changes.

The dispenser controller operates by forcing ink stored in a syringe through a nozzle using pulses of pressurised air. A vacuum is applied to the syringe when not printing to prevent ink being erroneously dispensed. The dispense pressure, dispense duration and vacuum pressure can be adjusted using the dispenser controller which is commercially available. The syringe or substrate is mounted on an XYZ moving stage depending on the setup. The $\mathrm{X}$ and $\mathrm{Y}$ stage movement is controlled to deposit ink in the desired area of the substrate. 
The $\mathrm{Z}$ stage is set to a defined $\mathrm{Z}$ height above the substrate before printing starts. Figure 2 shows a diagram of a typical dispenser printer and Figure 3 shows the bespoke dispenser printer used in this work with an optional curing head also attached next to the syringe.

When printing, the XY stages are continuously moved under the nozzle, which is subject to an applied air pressure for the duration of the print. When the print is complete, the syringe is then subject to an applied vacuum to prevent the ink continuing to be dispensed. For each ink the dispenser parameters are altered to adjust the volume of ink deposited onto an area. The key dispenser parameters are the nozzle movement speed, the dispensing pressure, the nozzle diameter and type, and the distance between adjacent printed lines. The distance between adjacent lines defines the spacing between printed lines which must be larger than the nozzle diameter as the ink will bleed causing discrete lines to coalesce into a uniform film.

\section{3) Printed Electroluminescent Lamps}

EL lamps are fabricated using a typical capacitor structure with an emitting phosphor layer sandwiched between the two electrodes. One of the electrodes is transparent to allow the light to be emitted. A dielectric layer is printed between the electrodes to prevent a short circuit since the phosphor layer would break down under the high field strengths. A total of four discrete layers are required for a printed EL lamp. The structure used for the EL lamps in this work is shown in Figure 4 below. The purpose of each layer and the ink used are described in Table 1 below. The Fabinks range of inks were selected as they are all tested for suitability to print onto fabric, and are designed to maintain the fabric's flexibility.

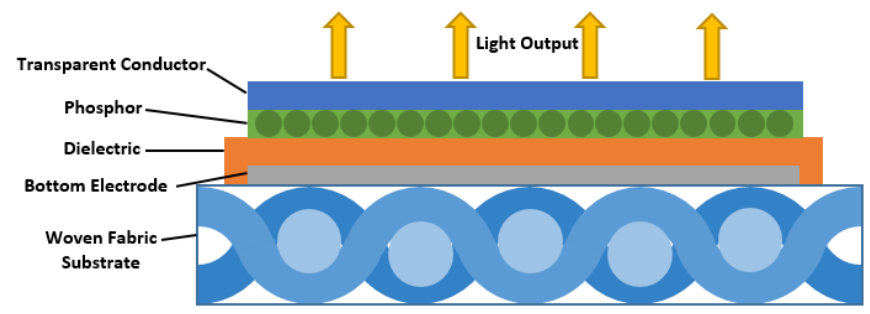

Figure 4. Structure of printed EL Lamp.

Table 1. A brief description of each printed layer with the ink used.

\begin{tabular}{|c|l|c|}
\hline $\begin{array}{c}\text { Layer } \\
\text { Name }\end{array}$ & \multicolumn{1}{|c|}{ Layer Description } & Ink Used \\
\hline $\begin{array}{c}\text { Bottom } \\
\text { electrode }\end{array}$ & $\begin{array}{l}\text { Provides one side of the capacitor structure } \\
\text { and evenly distributes the charge. }\end{array}$ & $\begin{array}{c}\text { FabInks } \\
\text { TC-C4001 }\end{array}$ \\
\hline Dielectric & $\begin{array}{l}\text { Prevents short circuits across the capacitor } \\
\text { structure and acts as a light reflector. }\end{array}$ & $\begin{array}{c}\text { FabInks } \\
\text { TC-D9001 }\end{array}$ \\
\hline Phosphor & $\begin{array}{l}\text { Emits light under the influence of a strong } \\
\left.\text { alternating electric field (2MVm }{ }^{-1}\right) .\end{array}$ & $\begin{array}{c}\text { FabInks } \\
\text { TC-P0001 }\end{array}$ \\
\hline $\begin{array}{c}\text { Transparent } \\
\text { Conductor }\end{array}$ & $\begin{array}{l}\text { Provides the top electrode across the } \\
\text { phosphor layer whilst allowing light to be } \\
\text { transmitted out from the phosphor layer. }\end{array}$ & $\begin{array}{c}\text { FabInks } \\
\text { TC-C4006 }\end{array}$ \\
\hline
\end{tabular}

Current commercial printed EL lamps are often made using screen printing and require a transparent conductor made from a glass or plastic substrate completely coated in Indium Tin Oxide (ITO) regardless of the shape of the lamp. ITO is usually sputtered onto the substrate and generally requires high temperature processing after deposition [14] making it unsuitable for use with fabrics which are not normally exposed to temperatures above $150^{\circ} \mathrm{C}$ to reduce possible chance of damage. In this work the transparent conductor ink is based on poly(3,4-ethylenedioxythiophene):poly(styrene sulfonate) (PEDOT:PSS). The bottom electrode ink is based on silver (Ag) particles, the dielectric layer is based on Barium Titanate (BaTiO3) particles, and the phosphor layer uses Zinc Sulfide doped with Copper and Aluminium ( $\mathrm{ZnS}: \mathrm{Cu}, \mathrm{Al})$. All of these particles are formulated into inks using flexible polymer binders.

The first printed layer in the structure shown in Figure 4 is a conducting layer which forms the bottom electrode of the capacitor structure. As no light passes through this layer, opaque conductors can be used. The conductivity of the material is important as it affects the homogeneity of the charge across the electrode which in turn controls whether a uniform visible light is produced. Typically silver inks are used due to their low resistivity $(<15 \mathrm{~m} \Omega / \mathrm{sq} / \mathrm{mil})$ and low temperature processing $\left(<130^{\circ} \mathrm{C}\right)$, although such inks are expensive and can suffer from electromigration [3]. An alternative is carbon ink which has the disadvantage of a relatively high resistivity $(20 \Omega / \mathrm{sq} / 25 \mu \mathrm{m})$.

The next printed layer in the structure shown in Figure 4 is a dielectric layer. Typically Barium Titanate (BaTiO3) inks have been used as a dielectric layer due to their high dielectric constant of 2000-3000 at room temperature [15]. The inks perform well with a particle size under $1 \mu \mathrm{m}$ [3] reducing the likelihood of the particles causing a blockage when printing.

Phosphor is usually printed as a separate layer on top of the dielectric. Almost all EL lamps utilise ZnS phosphors doped with acceptor/donor materials. Table 2 provides a summary of the common dopants and the emission colour they produce. The phosphor particles are mixed with a transparent polymer binder to create a printable ink.

Table 2. Table comparing common $\mathrm{ZnS}$ dopants against the visible emission colour they produce (data from [16]).

\begin{tabular}{|l|l|l|}
\hline Zns:Dopant & $\begin{array}{l}\text { Peak } \\
\text { Wavelength } \\
\text { (nm) }\end{array}$ & $\begin{array}{l}\text { Colour } \\
\text { Approximation }\end{array}$ \\
\hline $\mathrm{ZnS}: \mathrm{Cu}, \mathrm{Cl}$ & 450 & Blue \\
\hline $\mathrm{Zns}: \mathrm{Cu}, \mathrm{Al}$ & 540 & Green \\
\hline $\mathrm{ZnS}: \mathrm{Cu}, \mathrm{Mn}, \mathrm{Cl}$ & 590 & Green/Yellow \\
\hline $\mathrm{ZnS}: \mathrm{Cu}$ & 690 & Orange/Red \\
\hline
\end{tabular}

A printed transparent conductor forms the top electrode of the EL lamp; as with the bottom electrode, a high conductivity is important to distribute the charge evenly. As the light must pass through this layer, transmittance is also an important characteristic. A low transmittance reduces the perceived brightness and can also distort the colour if only part of the spectrum passes through the conductor. On non-fabric substrates ITO has been used for this layer, but as previously discussed, ITO usually requires high temperature processing 
making it unsuitable. ITO is also expensive making it unappealing for commercial applications. Conductive polymers are currently the only feasible alternative suitable for fabrics.

\section{4) Results and Discussion}

A woven $100 \%$ polyester fabric was selected as the substrate to print the EL lamps onto. It was selected as it is a readily available woven fabric making it representative of a number of other fabrics used in applications ranging from clothing to architecture. The fact that it is woven adds significant challenge as the weave effectively contains a network of voids that the inks must fill to give a uniform surface. The inks shown previously in Table 1 each had optimum dispensing parameters defined through a series of single line printing tests. A $250 \mu \mathrm{m}$ inside diameter tapered nozzle and a stage movement speed of $10 \mathrm{~mm} / \mathrm{s}$ was used for all layers. The nozzle was selected as the smallest diameter that printed reliably without blockages. The small diameter allows fine control over the size of the printed lines. The speed was selected as it was the maximum the moving stages could move without exceeding their repeatability tolerances. The dispense pressure and vacuum pressure used to print each layer is shown in Table 3 below.

Table 3. The dispense pressure and vacuum pressure used to print each of the layers in the dispenser printed EL lamp

\begin{tabular}{|c|c|c|}
\hline Layer Name & $\begin{array}{c}\text { Dispense Pressure } \\
\mathbf{( k P a )}\end{array}$ & Vacuum Pressure (kPa) \\
\hline Bottom electrode & 10 & 0.8 \\
\hline Dielectric & 40 & 0.4 \\
\hline Phosphor & 30 & 0.6 \\
\hline Transparent Conductor & 1.2 & 0.2 \\
\hline
\end{tabular}

The layers were printed consecutively and each cured for 10 minutes at $125^{\circ} \mathrm{C}$ in a box oven before the next layer was printed. Figure 5 shows each printed layer after curing. The initial bottom electrode layer printed well, with some minor bleeding visible along the right of the layer. The dielectric layer was then printed with a $1 \mathrm{~mm}$ overlap on the bottom electrode. $1 \mathrm{~mm}$ was chosen as it allows sufficient tolerance for misalignment of the printed layers and also any bleeding. This tolerance is important to preventa short circuit between the bottom electrode and transparent conductor. The phosphor layer printed well with no bleeding, followed by a bus electrode layer. This layer is a simple silver connector, printed to allow convenient connection to the transparent conductor, however this is not essential and the driving voltage could be connected directly to the transparent conductor. The last layer was the transparent conductor. The PEDOT:PSS ink used for this layer is challenging to print as it has a relatively high surface tension, and therefore does not coalesce as easily as the other inks. The print lines are more visible after curing leading to a non-uniform surface, this will affect the field distribution, which will in turn affect the light output. However the effect will not be significant, especially at higher driving voltages.

The lit printed EL can be seen being driven with a $150 \mathrm{~V}$,

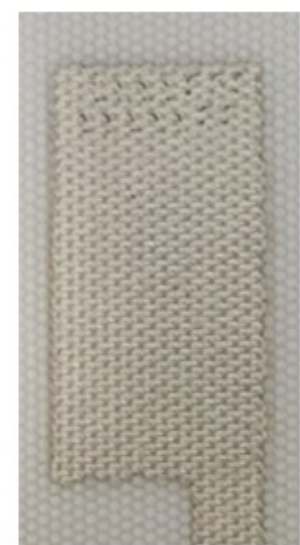

Bottom Electrode

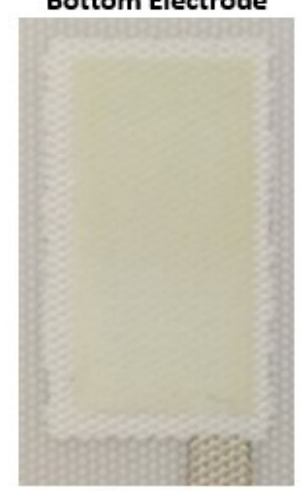

Phosphor

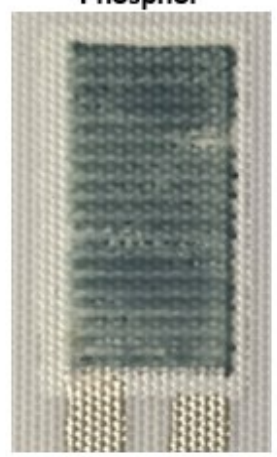

Transparent Conductor

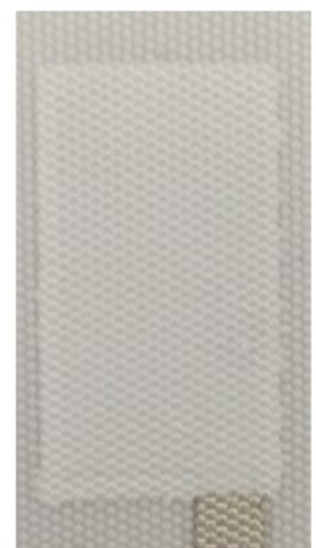

Dielectric

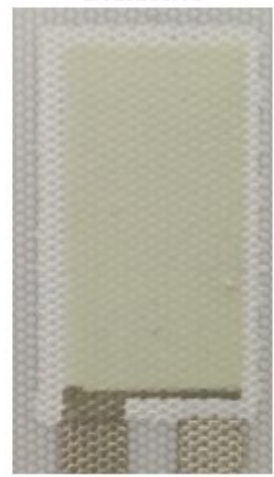

Bus Electrode

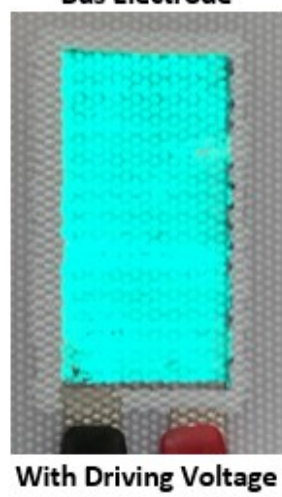

Figure 5. Images showing each printed layer in the EL lamp after curing, and an EL lamp being driven at $150 \mathrm{~V}, 400 \mathrm{~Hz}$.

$400 \mathrm{~Hz}$ signal in the last image in Figure 5. The nonuniformities in the light distribution are caused by one of two factors. The primary factor is variations in the transparent conductor layer thickness, which has a filtering effect on the light. Further variations in light distribution are caused by changes in field strength over the phosphor layer. The field strength is varied by the weave of the fabric altering the distance between the electrodes.

Each printed layer's thickness was measured using an SEM. A technique was developed where the samples are cooled in liquid Nitrogen for 15 minutes leaving them brittle. The samples were then fractured using a sharp blade to reveal the structure in the center of a lamp. This technique is preferred over simply cutting the samples as this crushes the printed layers resulting in poorly representative images. The results from the SEM revealed that layer thicknesses varied with the weave of the fabric, causing the variations in brightness seen in Figure 5. The SEM cross-section can be 


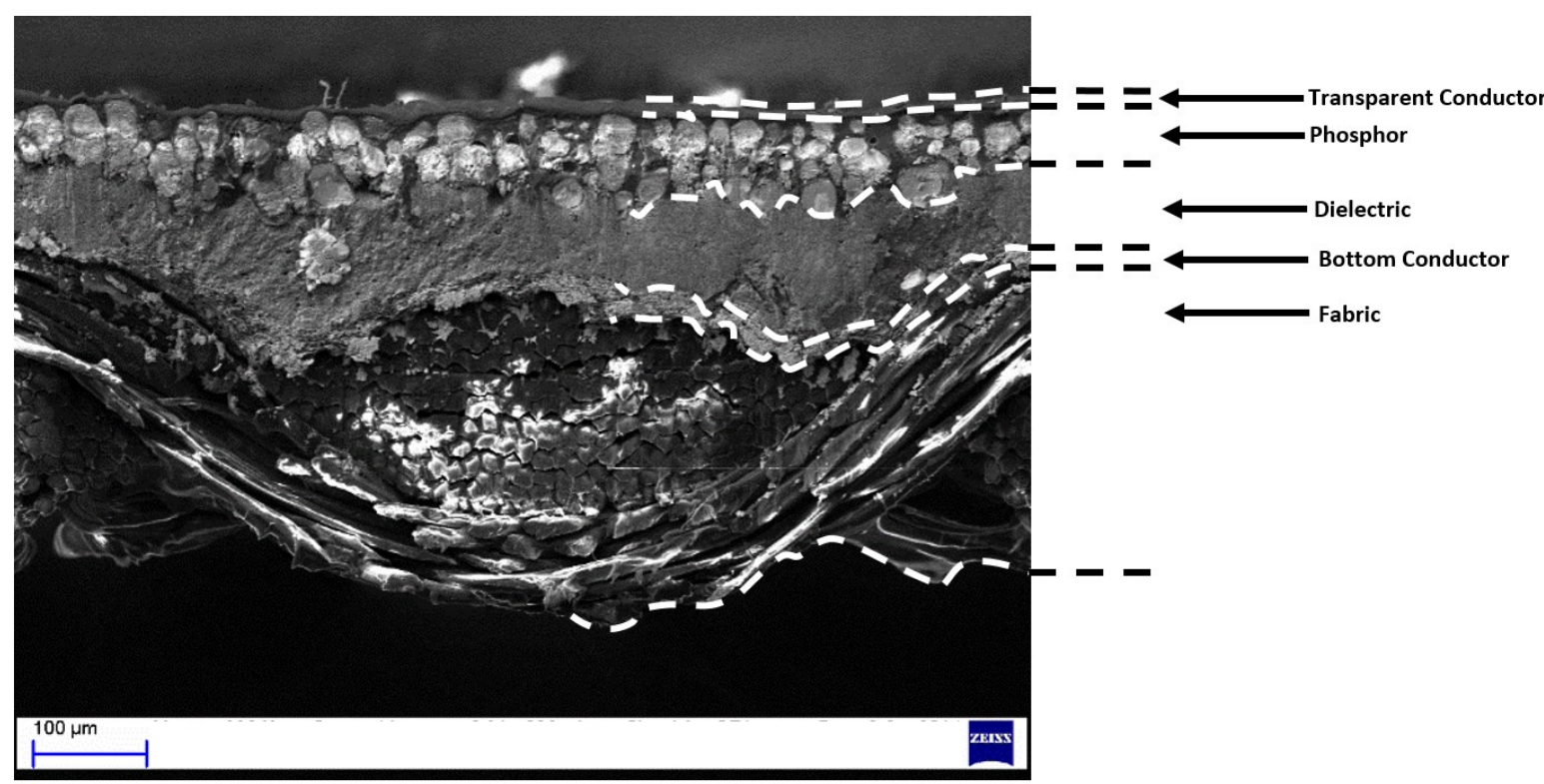

Figure 6. SEM cross-section image with the layer boundaries highlighted with a dashed line.

seen in Figure 6. Distinct layers are visible and it can be seen how the consecutive layers smooth the weave of the fabric to provide an overall smooth surface. Each layer thickness was measured using the SEM and the results are shown in Table 4, along with the target thickness for each layer.

The target thickness for the bottom conductor is based on achieving a continuous layer with all areas connected to each other. Below $10 \mu \mathrm{m}$ the dispenser printer is unable to reliably produce uniform layers due to the undulating surface of the fabric weave, therefore a target of $>10 \mu \mathrm{m}$ was set. The target thickness for the dielectric material is based on achieving a breakdown voltage in excess of $500 \mathrm{~V}$. The EL lamps will be tested at up to $400 \mathrm{~V}$ peak, therefore a minimum thickness of $30 \mu \mathrm{m}$ based on the material datasheet will give a breakdown voltage of $500 \mathrm{~V}$. $500 \mathrm{~V}$ was selected to give a small tolerance above the maximum testing voltage. The target thickness for the phosphor layer is based on achieving a layer with single phosphor particles side by side and a small amount of binder material to hold them in place. If multiple phosphor particles are vertically stacked then the field strength over them is reduced, and the emitted light from the lower phosphor particles is reabsorbed. The particles are an average diameter of $30 \mu \mathrm{m}$, therefore a target thickness of $40 \mu \mathrm{m}$ was selected to allow a small amount of binder material above and below the particles. The target thickness for the PEDOT:PSS was set using the same theory behind the bottom conducting layer minimum thickness. The layer should be as thin as possible with a homogeneous coverage, based on the limitations of the dispenser printer a target thickness of $>10 \mu \mathrm{m}$ was set.

The measured layer thicknesses closely match the target thickness for the bottom conductor and transparent conductor layers. The dielectric is significantly thicker than the minimum thickness. The single layer was printed thicker in an attempt to smooth the weave of the fabric and ensure that there was at least $30 \mu \mathrm{m}$ of dielectric ink in all areas to prevent the breakdown voltage being exceeded. The thicker dielectric layer will cause a reduced field strength to be present over the phosphor, resulting in a lower luminance. However, a $30 \mu \mathrm{m}$ layer must be present in all areas, which is difficult to achieve due to the high surface roughness $\left(R_{a}=42 \mu \mathrm{m}\right)$ of the woven fabric. The phosphor layer was also printed $33 \%$ thicker than the target thickness, this was caused by variations in the height of the underlying dielectric layer, meaning more ink was dispensed in some areas than others.

Where a significant variation in measured thicknesses occurred an average was taken from five thickness measurements. To reduce the variations in thickness of the printed layers an extra layer could be printed first to smooth the woven fabric surface, as used by Yang et al [17].

Table 4. Layer thickness measurements from SEM cross-section of EL lamp.

\begin{tabular}{|c|c|c|c|c|}
\hline Layer & $\begin{array}{c}\text { Bottom } \\
\text { Conductor }\end{array}$ & Dielectric & Phosphor & $\begin{array}{c}\text { Transparent } \\
\text { Conductor }\end{array}$ \\
\hline $\begin{array}{c}\text { Target } \\
\text { Thickness } \\
(\mu \mathrm{m})\end{array}$ & $>10$ & $>30$ & 40 & $>10$ \\
\hline $\begin{array}{c}\text { Measured } \\
\text { Thickness } \\
(\mu \mathrm{m})\end{array}$ & 12 & 97 & 53 & 14 \\
\hline
\end{tabular}

\section{5) Lamp Performance and Evaluation}

To quantify the brightness and colour of the dispenser printed EL lamps, a Konica Minolta CS-100 was used to record luminance and colour co-ordinate data, a similar technique was used by Wang et al. [8]. The lamps were driven using a Trek PZD700A high voltage amplifier with voltages from $50-400 \mathrm{~V}$ peak, and frequencies of $200 \mathrm{~Hz}, 400 \mathrm{~Hz}$, $1000 \mathrm{~Hz}$ and $2000 \mathrm{~Hz}$. The results can be seen in Figure 7 below. The graph shows the luminance increases with greater driving voltages, and also with higher driving frequencies as expected. 


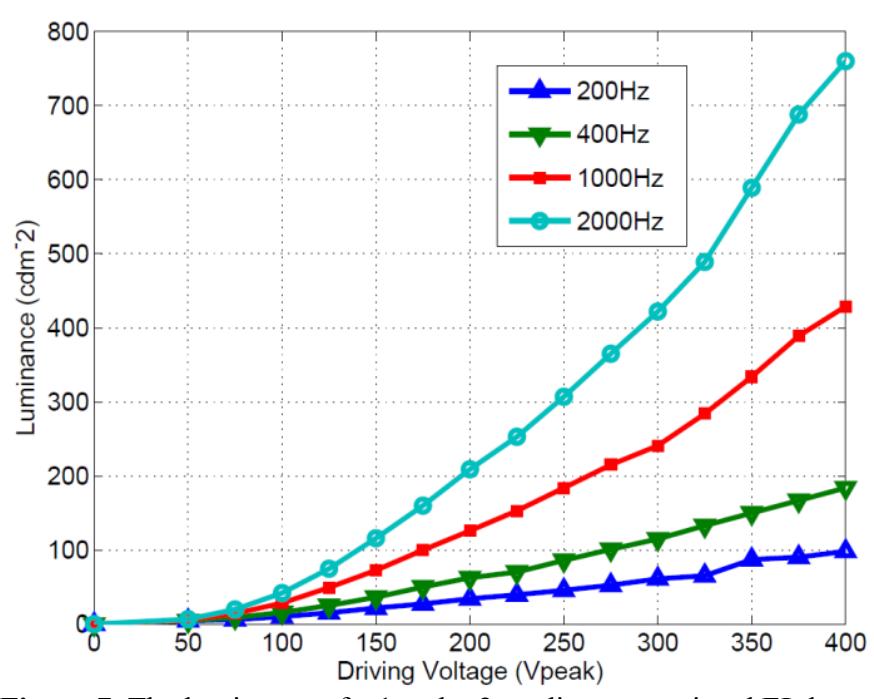

Figure 7. The luminance of a $1 \mathrm{~cm}$ by $2 \mathrm{~cm}$ dispenser printed EL lamp at various driving voltages and frequencies.

A colour shift based on the driving frequency was also seen during testing. The phenomenon has previously been exaplained by Jayaraj \& Vallabhan [18]. The colour shift is significant and could be useful in some design applications with colour offering a second level of communication (lamp being on or off being the first). The colour shift is shown on a CIE 1931 colour space chromacity diagram in Figure 8. The data shows a shift towards a lower wavelength of blue light at higher driver frequencies.

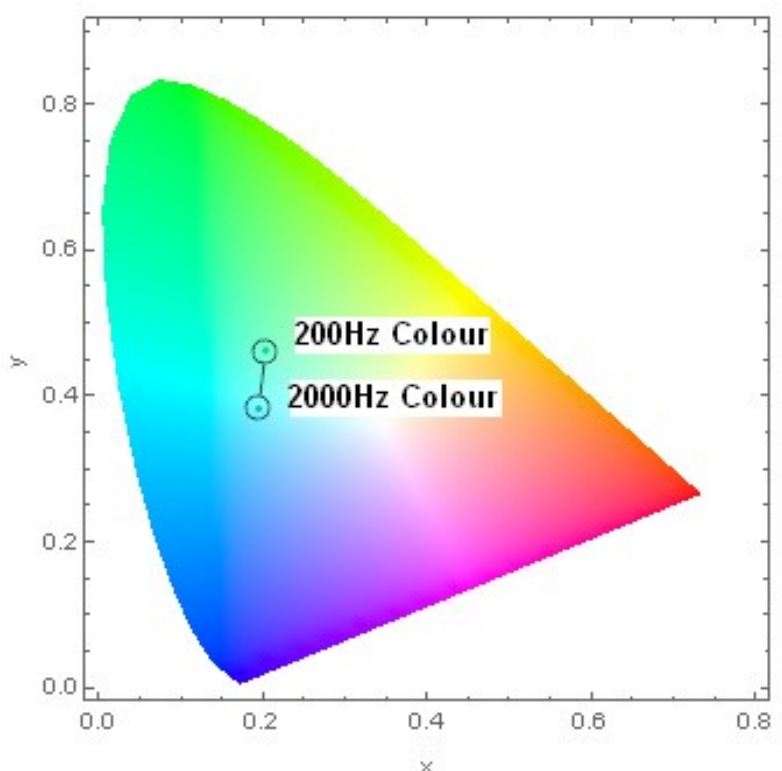

Figure 8. CIE 1931 colour space chromacity diagram showing the colour shift of a dispenser printed EL lamp based on driving frequency.

Screen-printed EL lamps were produced in the same dimensions to allow comparison to a commonly used printing technique. The lamps were fabricated using the same method as described in a previous publication [10]. The same inks were used for both the dispenser and screen-printed lamps and the same measurement technique was used to record the luminance data. A graph comparing the luminance data for the dispenser and screen-printed EL lamps over a range of driving voltages and frequencies can be seen in Figure 9. It is important to note that a direct comparison between the lamps should not be made as a number of uncontrolled factors affect the brightness of the lamps such as the layer thicknesses, print homogeneity, print roughness, and small pinholes in the layers. Instead the graph shows that dispenser printing is a viable technology for the production of EL lamps on fabric.

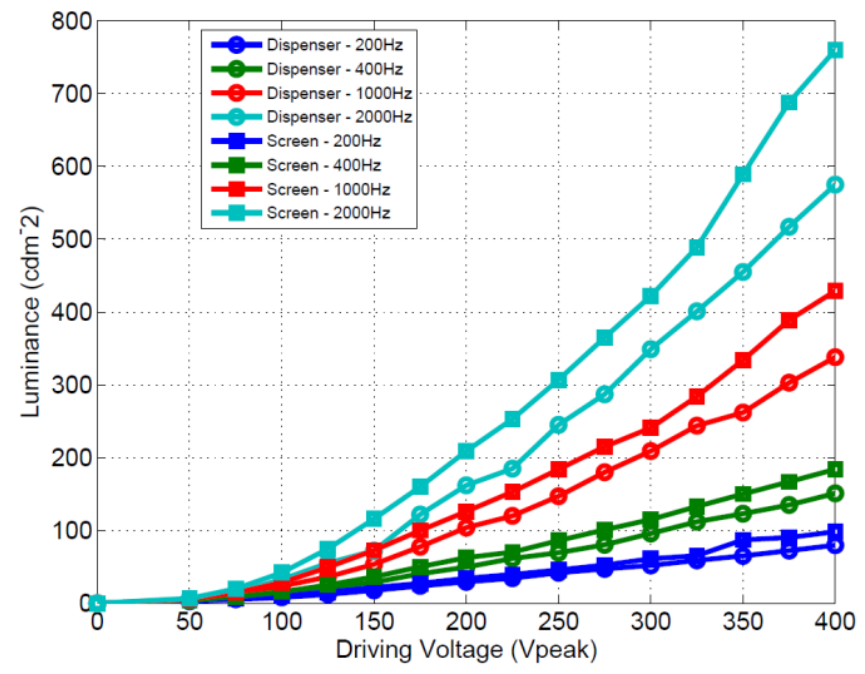

Figure 9. Comparison between a dispenser and screen-printed EL lamp both with dimensions $1 \mathrm{~cm}$ by $2 \mathrm{~cm}$ over a range of driving voltages and frequencies.

The results show that the screen-printed lamps are brighter - on average they have a $25 \%$ higher luminance, with an error of $\pm 5 \%$ due to variations in luminance over the EL lamp surface. The difference can be attributed to the screen-printer currently having finer control of layer thickness than the dispenser printer. This is visible when comparing the thickness of the dielectric layer, which is $64 \mu \mathrm{m}$ for the screen-printed EL lamp compared to $97 \mu \mathrm{m}$ for the dispenser printed EL lamp. The thinner dielectric layer will result in a stronger field strength over the phosphor particles for a given drive voltage, causing a higher luminance.

The printed EL lamps on fabric were compared to a range of commercially available EL lamps. These commercial lamps are all screen-printed onto a polyurethane film, avoiding issues surrounding surface roughness of the substrate. The lamps tested were a range of sizes as shown on the graph legend, however the luminance is related to the field strength, which is the same for all of the lamps, therefore they are comparable. The light distribution is approximately uniform across the surface of the lamp, however three measurements for each voltage were taken and the measurements were then averaged.

The lamps were tested at $400 \mathrm{~Hz}$ as it is the most commonly used driving frequency for commercial EL drivers. The Trek PZD700A amplifier used earlier is capable of driving all sizes of EL lamps so this was used again. The results are shown in Figure 10 below, all sizes in the legend are in inches.

The results show that the two UoS fabric printed lamps are brighter than any of the commercial EL lamps. The University of Southampton (UoS) dispenser printed EL lamp has a luminance 1.5 times higher on average than the best of the commercial EL lamps. The higher luminance is likely to be caused by an improved print quality in laboratory conditions 
relative to a commercial production line and superior inks used to produce the lamps.

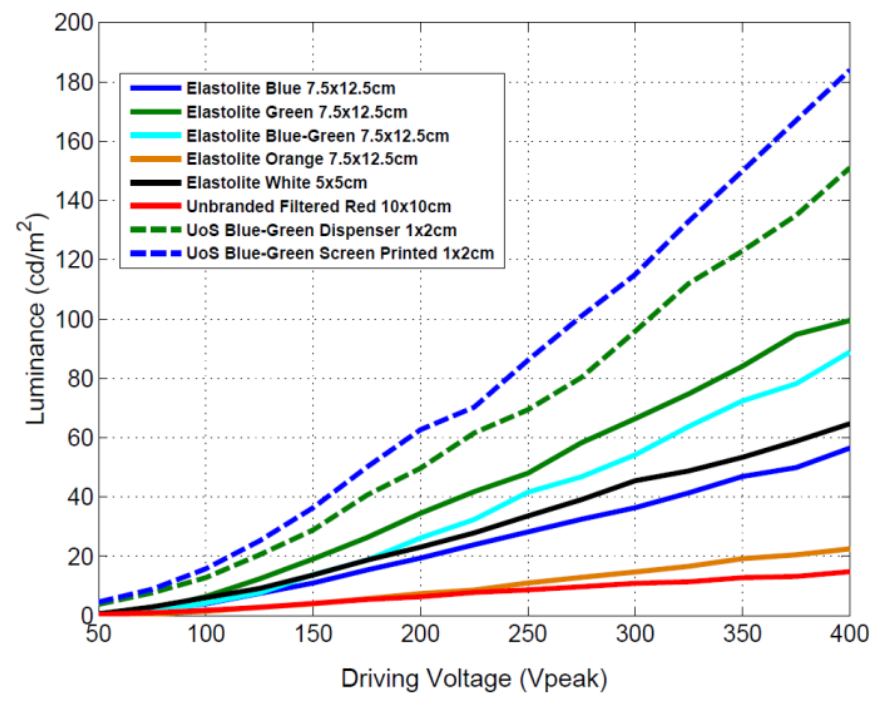

Figure 10. Comparison of UoS dispenser and screen printed EL lamps, with a range of commercial EL lamps. All sizes in the legend are in inches.

\section{6) Conclusions}

Dispenser printing is aimed at prototyping and research applications and bespoke designs, with other technologies, such as screen-printing, better suited to mass production. The luminance of the dispenser printed devices was shown to be comparable to those which are screen-printed, with screenprinting being an average of $25 \%$ higher. The UoS printed fabric EL lamps had a higher luminance than any of the commercial lamps on polyurethane that were tested. While a direct comparison is not possible due to the uncontrolled factors, it does show that dispenser printing is a viable fabrication technology for EL lamps.

The advantages of dispenser printing such as an easily changeable design, combined with its comparable performance when printing an EL lamp, make it an excellent fabrication technology. This work represents the first use of a dispenser to entirely fabricate an EL lamp. The capability of the printer has further been shown by printing the device onto fabric, which represents a challenging substrate due to the high surface roughness and absorption.

\section{7) Acknowledgements}

This research is part of the Framework Program 7 EU project CREATIF, funded by the European Commission under the 'Technologies and Scientific Foundations in the field of Creativity' theme in the 7th Framework Programme for Research and Technological Development.

\section{8) References}

[1] L. V. Langenhove and C. Hertleer, "Smart clothing: a new life," International Journal of Clothing Science and Technology, vol. 16, pp. 63-72, 2004.
[2] T. Ireland and J. Silver, "Studies on the Orientation of ACEL ZnS: Cu Particles in Applied AC Fields," ECS Journal of Solid State Science and Technology, vol. 3, pp. R25-R32, 2014.

[3] M. Bredol and H. Schulze Dieckhoff, "Materials for powder-based AC-electroluminescence," Materials, vol. 3, pp. 1353-1374, 2010

[4] P. D. Rack and P. H. Holloway, "The structure, device physics, and material properties of thin film electroluminescent displays," Materials Science and Engineering: R: Reports, vol. 21, pp. 171-219, 1998. [5] J. Burroughes, D. Bradley, A. Brown, R. Marks, K. Mackay, R. Friend, et al., "Light-emitting diodes based on conjugated polymers," nature, vol. 347, pp. 539-541, 1990.

[6] C. W. Tang and S. A. VanSlyke, "Organic electroluminescent diodes," Applied physics letters, vol. 51, pp. 913-915, 1987.

$\mathrm{X}$. Tao, Wearable electronics and photonics: Elsevier, 2005

J. Wang, C. Yan, K. J. Chee, and P. S. Lee, "Highly Stretchable and Self-Deformable Alternating Current Electroluminescent Devices," Advanced Materials, vol. 27, pp. 2876-2882, 2015.

[9] Y. Li, R. Torah, S. Beeby, and J. Tudor, "Inkjet printed flexible antenna on textile for wearable applications," in Textile Institute World Conference, Selangor, Malaysia, 2012.

[10] M. de Vos, R. Torah, S. Beeby, and J. Tudor, "Functional Electronic Screen-printing Electroluminescent Lamps on Fabric," in Eurosensors, 2014, pp. 1513-1516.

[11] M. de Vos, R. Torah, and J. Tudor, "A Novel Pneumatic Dispenser Fabrication Technique for Digitally Printing Electroluminescent Lamps on Fabric," in DTIP 2015, Montpellier, France, 2015.

[12] P. Calvert, "Inkjet printing for materials and devices," Chemistry of materials, vol. 13, pp. 3299-3305, 2001.

[13] P. D. Angelo, "Inkjet-printed light-emitting devices: applying inkjet microfabrication to multilayer electronics," University of Toronto, 2013.

[14] S.-J. Hong, J.-W. Kim, and J. I. Han, "Improvement of electrical properties of printed ITO thin films by heat-treatment conditions," Current Applied Physics, vol. 11, pp. S202-S205, 1// 2011.

[15] G. Arlt and D. Hennings, "Dielectric properties of fine-grained barium titanate ceramics," Journal of Applied Physics, vol. 58, pp. 1619-1625, 1985.

[16] Y. A. Ono, Electroluminescent displays vol. 1: World Scientific, 1995.

[17] K. Yang, R. Torah, Y. Wei, S. Beeby, and J. Tudor, "Waterproof and durable screen printed silver conductive tracks on textiles," Textile Research Journal, vol. 83, pp. 2023-2031, November 1, 2013 2013.

[18] M. Jayaraj and C. Vallabhan, "A study of the white electroluminescence in $\mathrm{ZnS}: \mathrm{Cu}, \mathrm{Pr}, \mathrm{Cl}$ phosphors," Journal of Physics D: Applied Physics, vol. 22, p. 1380, 1989. 